\title{
Nanomedicine for the reduction of the thrombogenicity of stent coatings
}

This article was published in the following Dove Press journal:

International Journal of Nanomedicine

26 March 2010

Number of times this article has been viewed

\section{Varvara C Karagkiozaki ${ }^{1,2}$ \\ Stergios D Logothetidis ${ }^{2}$ Spyridon N Kassavetis ${ }^{2}$ George D Giannoglou'}

'Aristotle University of Thessaloniki, Medical School, AHEPA University General Hospital, Ist Cardiology Department, Cardiovascular Engineering and Atherosclerosis Laboratory, Greece; ${ }^{2}$ Aristotle University of Thessaloniki, Physics Department, Laboratory for Thin Films - Nanosystems and Nanometrology (LTFN), Greece
Correspondence: Stergios Logothetidis Professor in Physics, Aristotle Department of Physics, Director of Laboratory for Thin Films - Nanosystems and Nanometrology (LTFN), Aristotle University of Thessaloniki, 54124 , Greece

Tel +302310998174

$\mathrm{Fax}+302310998390$

Email logot@auth.gr

\begin{abstract}
The treatment of patients with drug-eluting stents (DES) continues to evolve with the current emergence of DES technology that offers a combination of pharmacological and mechanical approaches to prevent arterial restenosis. However, despite the promising shortterm and mid-term outcomes of DES, there are valid concerns about adverse clinical effects of late stent thrombosis. In this study, we present an example of how nanomedicine can offer solutions for improving stent coating manufacturing, by producing nanomaterials with tailored and controllable properties. The study is based on the exploitation of human platelets response towards carbon-based nanocoatings via atomic force microscope (AFM). AFM can facilitate the comprehensive analysis of platelets behavior onto stent nanocoatings and enable the study of thrombogenicity. Platelet-rich plasma from healthy donors was used for the real-time study of biointerfacial interactions. The carbon nanomaterials were developed by $r f$ magnetron sputtering technique under controllable deposition conditions to provide favorable surface nanotopography. It was shown that by altering the surface topography of nanocoatings, the activation of platelets can be affected, while the carbon nanocoatings having higher surface roughness were found to be less thrombogenic in terms of platelets adhesion. This is an actual solution for improving the stent coating fabrication.
\end{abstract}

Keywords: stents nanomedicine, carbon coating, atomic force microscopy platelets nanotechnology

\section{Introduction}

Coronary drug-eluting stents (DES), being the new gold standard for percutaneous coronary revascularization, offer a benefit in reducing the acute vessel closure, restenosis and the need for repeat hospitalization, heart catheterizations and bypass surgery. A critical reassessment of the published evidence, however, suggests that the putative superiority of intravascular DES is founded on a questionable premise. ${ }^{1}$ Specifically, late stent thrombosis ( $>30$ days post procedure) in patients who receive DES is a primary area of interest because of the potential for serious adverse outcomes such as myocardial infarction and sudden death in an estimated $45 \%$ of these cases. ${ }^{2}$ This effect has been reported to occur at a rate of $0.2 \% /$ year in clinical trials ${ }^{3}$ and $0.6 \% /$ year with off-label use of DES in clinical practice. ${ }^{4}$

A possible higher rate of late thrombosis of the DES in comparison with bare metal stents has been shown recently. Indeed, nonclinical trial data show higher DES late thrombosis rates than those observed with bare metal stents. ${ }^{5}$ One of the possible mechanisms of DES late thrombosis is suggested to be the platelets activation and inflammatory reaction of the vessel wall associated with DES, either due to the 
polymers, the drug coatings themselves, or perhaps both. From a histological perspective, although DES can halt smooth muscle cells migration and proliferation owing to their antiproliferative drug elusion, in some cases they impair the re-endothelalization process with localized thrombus formation as observed with angiography or on histopathologic specimen. ${ }^{6}$ Rare occurrence of an eosinophilic reaction was described that might have led to late stent thrombosis and caused negative clinical outcomes. ${ }^{7}$

Besides the more widespread "off-label" use of DES and of the early discontinuation of antiplatelet therapy, different stent properties can subtly affect stent function, including modular design, metal coverage, strut thickness, strut shape, surface smoothness, coating materials, and drug properties. ${ }^{8}$ In addition to microscale stent surface features, the nanoscale surface texture, tailored through the deposition conditions of the nanocoatings, may be useful for improving stent function, given that the surface topography can promote vascular smooth muscle cell and endothelial cell adherence and proliferation. ${ }^{9,10}$ Several such coatings have been generated using a sol-gel process, in which a colloidal suspension (sol) of metal or ceramic is applied to a surface by dipping or spraying and subsequently bonded to form a porous, highly textured coating, such as titanium dioxide. Nanotextured coatings fit into a category of design concept that enhance endothelialization of stent struts and may reduce late thrombosis. ${ }^{11}$ Thus nanotechnology, an emerging multidisciplinary field of science that manufactures, studies and manipulates matter on a nanoscale, deals with the surface optimization of stents, by designing and developing biocompatible nanocoatings, as the first-layer cell formation on the surface is essential to minimize blood clotting. Nano approaches will allow the synthesis and control of materials in nanometer dimensions, and the in-depth analysis of bio/non-bio interactions via imaging nanotools providing access to new materials properties and stent characteristics in unprecedented ways. ${ }^{12}$ Polymeric drug barriers for stents are considered to have drawbacks due to the delay in endotheliazation of stent surface, which causes inflammatory reaction of the arterial wall. Therefore much research is being done on organic and inorganic thin films such as carbon-based coatings for artificial heart valves, blood pumps and stents. ${ }^{13,14}$

Here we describe the manufacture of two types of carbon nanocoatings using $r f$ magnetron sputtering deposition technique with variation in surface roughness as the main variable for the control of platelets response. An atomic force microscope (AFM) was used in order to investigate the thrombogenicity of the amorphous hydrogenated carbon
$(\mathrm{a}-\mathrm{C}: \mathrm{H})$ thin films and the role of tuning the deposition conditions for favorable surface nanostructure to inhibit thrombosis.

\section{Methods}

To manufacture the biomaterials, amorphous hydrogenated carbon nanocoatings, $80 \mathrm{~nm}$ thick, were deposited on silicon and stainless steel wafers by radio frequency reactive magnetron sputtering in a high vacuum chamber, in $\mathrm{Ar} / \mathrm{H}_{2}$ atmosphere by varying the hydrogen content (from $5 \%$ to $20 \% \mathrm{H}_{2}$ ). Two types of carbon thin films were developed under different deposition conditions: type A carbon nanocoatings with the application of ion bombardment on the substrate during deposition and type B carbon nanocoatings, without applying ion bombardment.

For the cell adhesion study, human platelet rich plasma (PRP) was prepared after the centrifugation of whole blood at $800 \mathrm{rpm}$ for 7 to 10 minutes at room temperature (RT). The blood was drawn by venopuncture from healthy donors who did not take aspirin or clopidogrel or other drugs that affect platelets function. Then, the PRP was diluted with homologous plasma at a ratio of 1:1000. The a-C:H nanocoatings were cleaned by $\mathrm{N}_{2}$ flow to remove any contaminants and incubated in PRP at RT. Then they were examined by AFM, after fixation of platelets with $5 \mu \mathrm{L}$ glutaraldehyde $1 \%$ at 1- and 2-hour intervals in order to acquire an AFM image of their morphology at a specific time. The SOLVER P47H scanning probe microscope (NT-MDT) was implemented in ambient environment, and to avoid platelets destruction by the sharp conical tip of AFM, the semi contact/tapping scanning mode of AFM was employed. AFM is a high-resolution microscopy, with demonstrated resolution of fractions of a nanometer, more than 1000 times better than that of optical microscopy, which is restricted by the optical diffraction limit. Unlike other imaging techniques such as scanning electron microscopy (SEM), this nanotool enables the biological entities-materials interactions to be investigated with nanoscale precision. ${ }^{15}$ While an electron microscope needs an expensive vacuum environment for proper operation, AFM can work perfectly well in ambient air or even a liquid environment, enabling cells to be studied in their near native environment without the need for special preparation, and guarantees cell viability. ${ }^{16}$ It can also provide size characterization in all three spatial dimensions, because it provides direct information about the height as well as lateral dimensions of the examined biological samples.

The AFM probe is a microscale cantilever with a sharp tip at its end (nominal tip radius $\sim 10 \mathrm{~nm}$ ) which is used to 
scan the specimen surface. An ionic repulsive force from the surface applied to the tip bends the cantilever upwards, and the amount of bending, measured by a laser spot reflected on to a split photo detector, can be used to calculate the force. By keeping the force constant while scanning the tip across the surface, the vertical movement of the tip follows the surface profile and the surface topography is acquired. In semi-contact scanning mode used in this study, the cantilever vibrates and thus taps the sample surface point after point without causing any destruction. ${ }^{17}$

The morphology of the adherent platelets, as an index of their activation, was investigated at 1- and 2-hour intervals and 10 fields were chosen at random to obtain statistical averages of the adherent platelets (by Student's $t$-test). The quantities used for the evaluation of surface roughness of the bare films and during platelets adhesion were peak to peak distance (peak-to-peak), and root-mean-square roughness $\left(\mathrm{R}_{\mathrm{rms}}\right)$. Quantitative and qualitative data provided from AFM imaging are highly informative for evaluating thrombogenicity of the case-study materials, whose selection was based on the fact that the carbon-based materials with an increased fraction of $\mathrm{sp}^{3}$ bonds are known to possess high mechanical hardness, chemical inertness and low friction coefficient, and have also shown good blood compatibility. ${ }^{18}$ In our previous work, we deduced that a-C:H thin films with $42 \% \mathrm{sp}^{3}$ hybridized carbon bonds content and a small amount of $\mathrm{H}_{2}$ in plasma during deposition exhibit good hemocompatibility on the scope of protein adsorption. ${ }^{19,20}$ In order to validate the AFM results, complementary SEM measurements with a $20 \mathrm{kV}$ JEOL 840A SEM were made to visualize platelets on type $A$ and type $B$ carbon nanocoatings with $5 \% \mathrm{H}_{2}$ in plasma during deposition after 1 hour of incubation.

As proteins and cells determine biomaterials thrombogenicity, these results create a basis for investigating further the interfacial phenomena of platelets interactions with the selected carbon nanocoatings.

\section{Results}

The implantation of biomaterials into the human body is followed by a surge of proteins and cells to cover their surface, and bioreactions with the surrounding biological tissue, which are governed by the implant surface. The first major event after a medical device comes in contact with blood is the adsorption of plasma proteins that tune the cell adhesion and behavior. ${ }^{21}$ Platelets adhesion followed by aggregation and spreading is the prerequisite event for thrombus formation, which impairs the implant's length of life. For vascular stents, heart valves and pumps that come in direct contact with blood, platelets behavior is a key issue for the evaluation of hemocompatibility of biomaterials. This study focuses on the morphological changes and interactions of human platelets with nanomaterials (a-C:H nanocoatings) probed by AFM.

At first, for AFM characterization of platelets adhesion on to the selected carbon nanocoatings, it should be mentioned that the platelets are disc shaped, and 0.5 to $3 \mu \mathrm{m}$ in size. After they come in contact with an artificial material and become activated, their shape becomes flatter, their granules are gathered into the center of the cell contributing to the composition of pseudo nucleus ('egg-like' type), and broad pseudopodia are extended. The elaboration of filopods made by the assembly of new cytoplasmic actin filaments is essential for their spread on to surfaces and their aggregation. After platelet adhesion, the platelet release reaction takes place in the adhering platelets, releasing serotonin, thromboglobulin, platelet factor IV, thromboxane $\mathrm{A}_{2}$, which leads to platelet aggregation on the surface. ${ }^{22,23}$ The previously mentioned morphological alterations of platelets during activation were taken into consideration for the analysis of the AFM images. Figures 1 and 2 show the stages of platelets activation. More precisely, in Figure 1, the platelet was activated after 15 minutes adhesion on carbon-based thin film and it lost its discoid shape and became spherical. Figure 2 shows highly activated platelets with filopods connected with each other forming a network, compromising a pre-stage of a thrombus. Figure 3 a shows an AFM image of activated platelets with pseudopodia on carbon nanocoatings after 1 hour of incubation. For cell adhesion studies, AFM, being superior to other imaging methods, can provide information with nanoscale precision, as indicated, for instance, by performing an arbitrary section of one activated platelet and measuring the height of its periphery with no filopods $(170 \mathrm{~nm})$, of its centre $(220 \mathrm{~nm})$ and its pseudopodia (100 nm) (Figure 3b).

By observing the AFM images of platelets adhering on to the selected carbon nanocoatings, it can be deduced that on the type $\mathrm{B}$ carbon thin films with $5 \% \mathrm{H}_{2}$ in plasma during deposition, after 1 and 2 hours incubation, the platelets undergo the following alterations in morphology during activation: i) loss of discoid shape, ii) extension of pseudopodia, iii) egg-like type formation, but without any development of aggregation (Figures $4 \mathrm{a}-\mathrm{b}$ ). In contrast, on type A carbon nanocoatings, it can be easily noticed in Figure 5a., that after 1 hour of incubation, platelets appear with a 'pseudo nucleus' in their center, being a index of activation, and after 2 hours they aggregate, forming a cluster look like an 

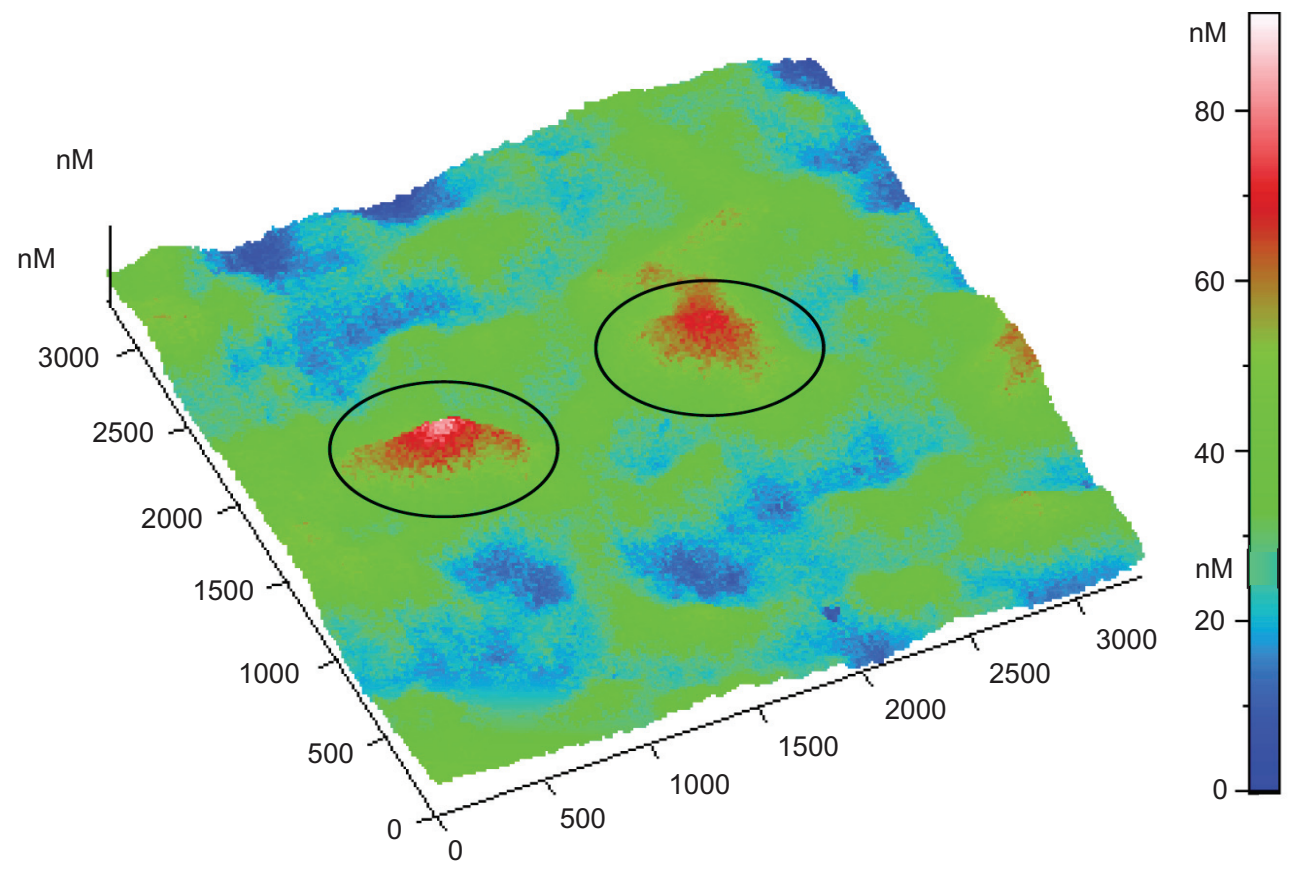

Figure I Three-dimensional AFM topography image of platelets (in circles) on a-C:H nanocoating after 15 minutes of incubation, at an early stage of activation.

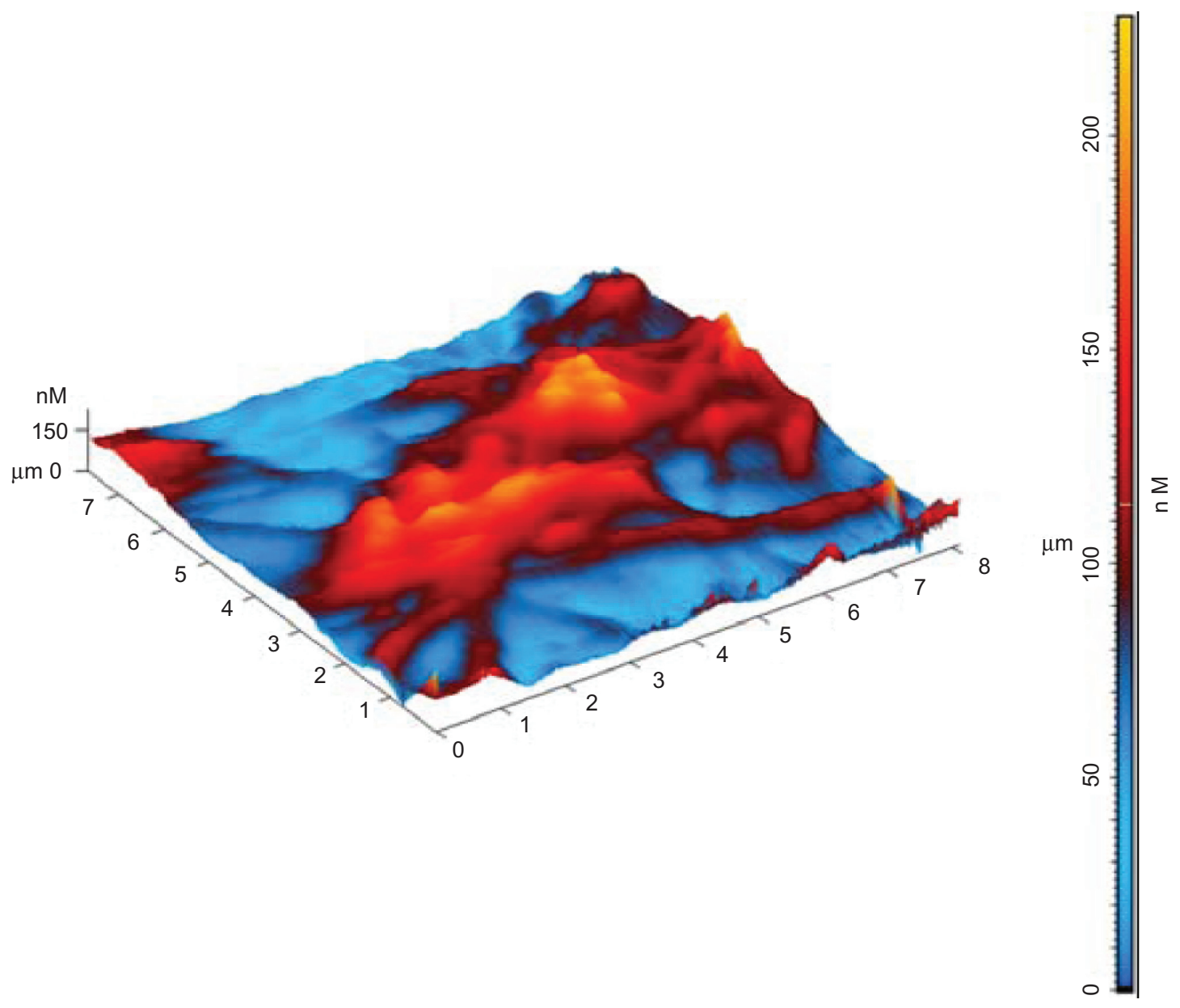

Figure 2 Three-dimensional AFM topography image of activated platelets on a-C:H nanocoating after 2 hours of incubation, interconnected with pseudopodia. 
A

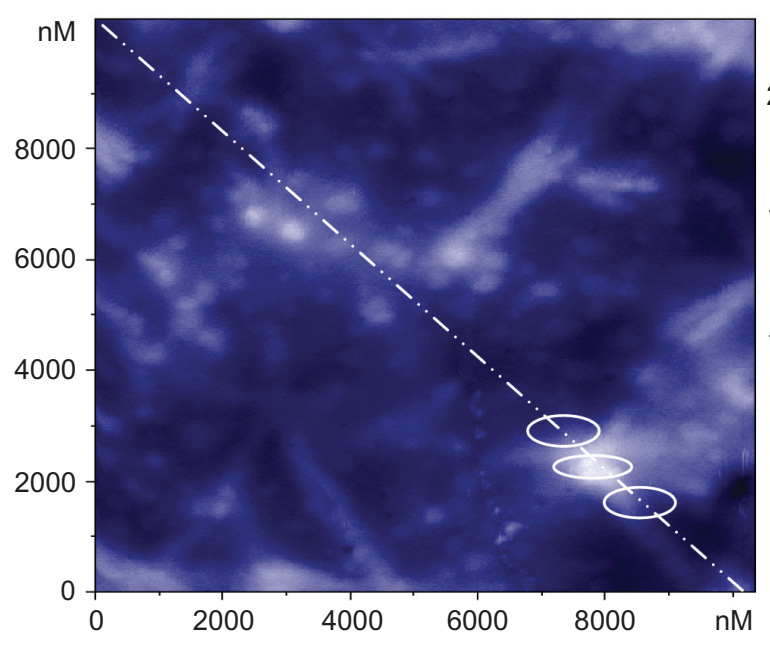

B

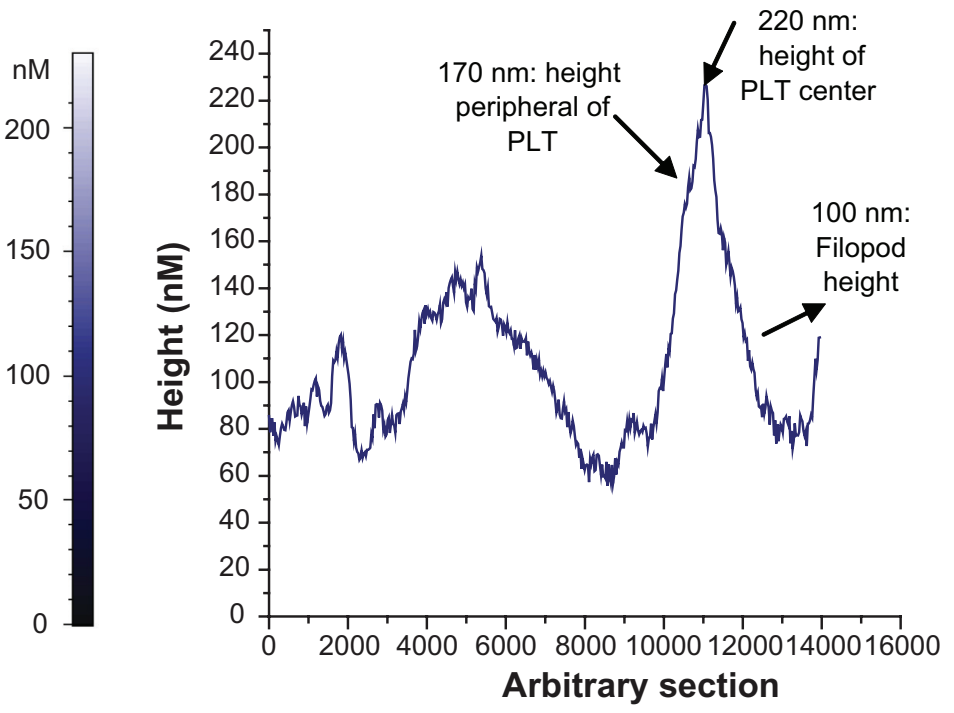

Figure 3 A) Two-dimensional AFM topography image of activated platelets with pseudopodia on a-C:H nanocoating after I hour of incubation; B) Arbitary section (white line) in $3 \mathrm{~A}$, of one activated platelet (PLT) at three points (white circles) with measurements of its components.

'island' (Figure 5b). It can be easily seen that platelets have a higher tendency to aggregate on the type $\mathrm{A}$ than on type $\mathrm{B}$ carbon nanocoatings. If the content of $\mathrm{H}_{2}$ in plasma during deposition of type A nanocoatings is increased up to $20 \%$, the observations of platelet AFM studies reveal that after 1 and 2 hours of incubation, the platelets undergo morphological changes during activation and they form clusters as well with a greater height of more than $1000 \mathrm{~nm}$ (Figures 6a-b). The higher content of hydrogen in the plasma is associated with the AFM observations that the platelets tend to make higher clusters on their surfaces. These data derived from AFM observations were confirmed by the measurements of surface roughness of the biofilms. The comparative diagram of a time-dependent increase of peak-to-peak and root mean
A

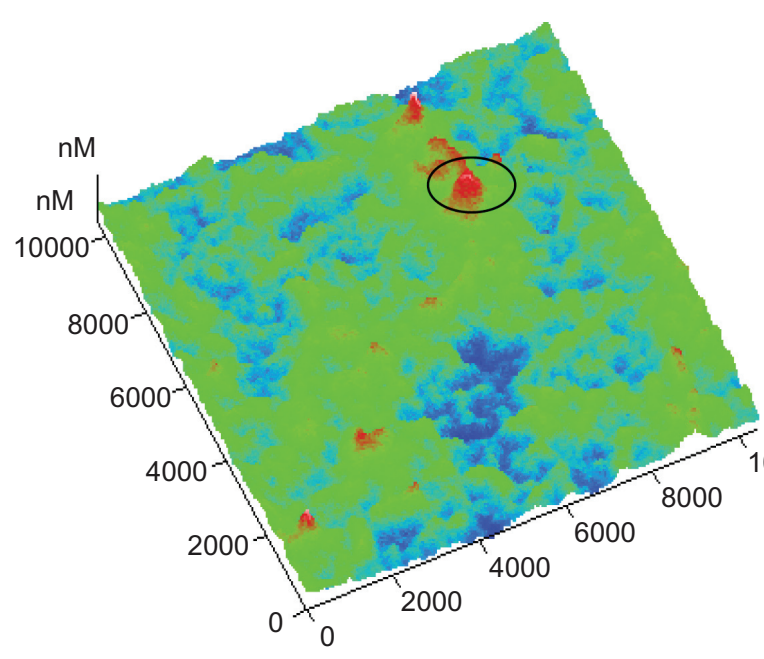

B

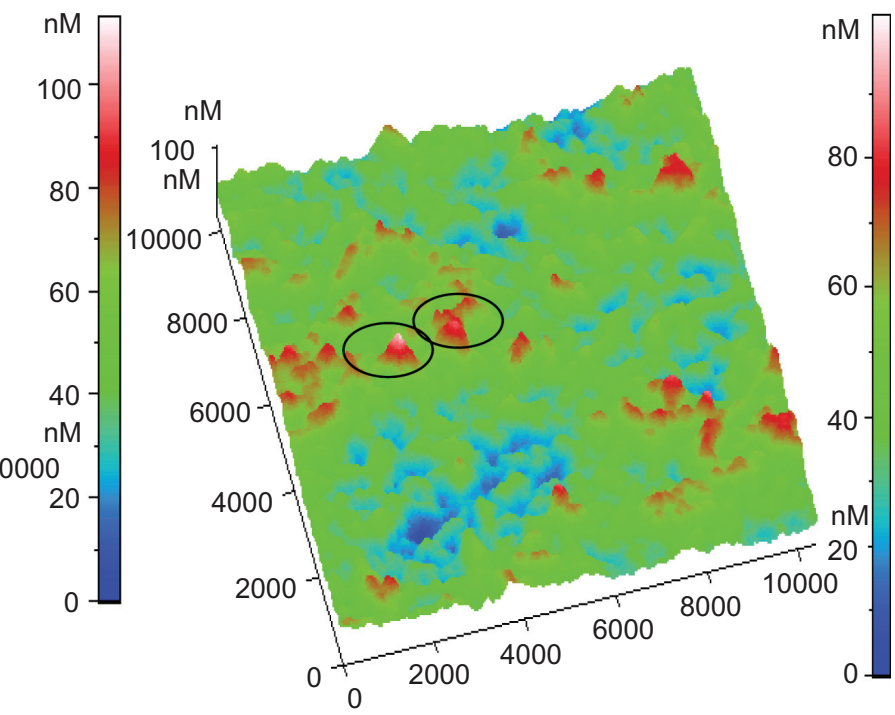

Figure 4 Three-dimensional AFM topography image of: A) Platelets on type B carbon nanocoating (after I hour incubation (scan size $10 \mu \mathrm{m} \times 10 \mu \mathrm{m}$ ). The circle indicates an activated platelet having the egg-like type structure; B) Platelets on type B carbon nanocoating after 2 hours incubation time (I0 $\mu \mathrm{m} \times 10 \mu \mathrm{m})$. The circles denote the egg-like type activated platelets. 
A

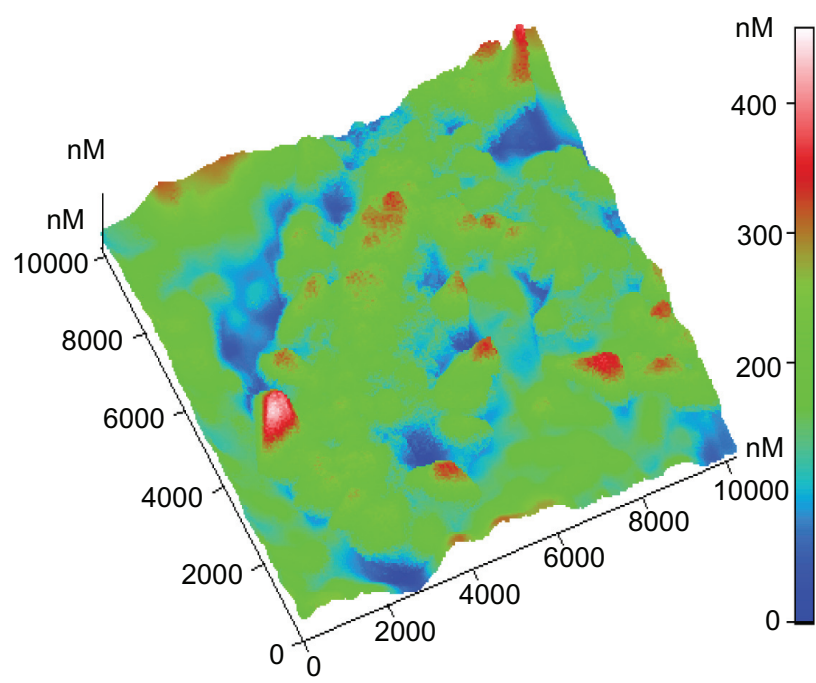

B

Figure 5 Three-dimensional AFM topography image of: A) Platelets on type A carbon nanocoating with $5 \% \mathrm{H}_{2}$ in plasma during deposition, after I hour of incubation (scan size $10 \mu \mathrm{m} \times 10 \mu \mathrm{m}$ ), B) Platelets on the same coatings as in Figure $5 \mathrm{~A}$, after 2 hours incubation time (scan size $21 \mu \mathrm{m} \times 21 \mu \mathrm{m}$ ). The arrows indicate the platelets aggregation and the formation of a cluster-like island.

square roughness parameters (Figure 7) and is indicative of platelets' gradual aggregation during time.

As a combination of parameters influence the response of blood components towards biomaterials, the nanotopography and surface roughness of the examined films were assessed to correlate with their thrombogenicity. The peak-to-peak and $\mathrm{R}_{\mathrm{rms}}$ values of the bare a-C:H nanocoatings (without the platelets) are shown in Table $1(P<0.001)$ type A carbon nanocoatings exhibit lower surface roughness than the type B nanocoatings, which can be attributed to the $\mathrm{Ar}^{+}$ion bombardment of the growing nanocoating during deposition. In order to validate our results, SEM measurements were made for the visualization of platelets conformation on type $\mathrm{A}$ and $\mathrm{B}$ carbon nanocoatings with $5 \% \mathrm{H}_{2}$ in plasma during deposition after 1 hour of incubation (Figure 8).

An inactivated platelet with spherical shape is presented in Figure 8a. As can be seen in Figure 8b, the platelets on type B carbon nanocoatings (with $5 \% \mathrm{H}_{2}$ in plasma during deposition) remain inactivated as they do not form pseudopodia or clusters, whereas on the type A nanocoatings, the cells
A

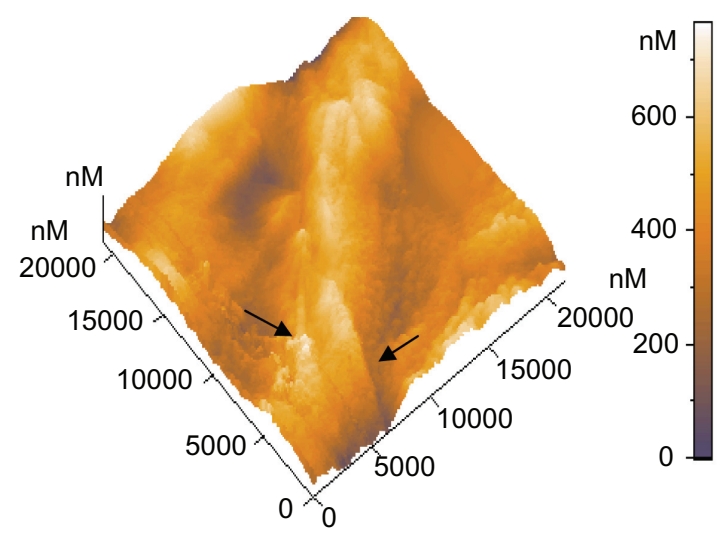

B

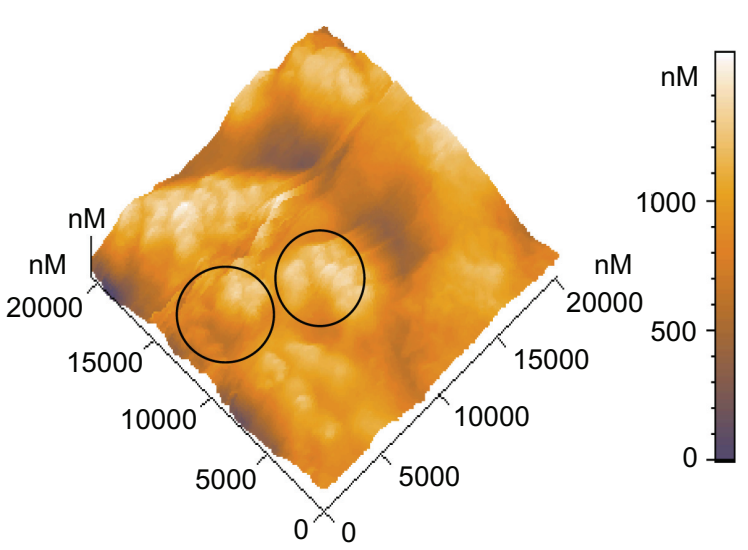

Figure 6 Three-dimensional AFM topography image of: A) Platelets on type A carbon nanocoatings with $20 \% \mathrm{H}_{2}$ in plasma during deposition after I hour incubation (scan size $21 \mu \mathrm{m} \times 21 \mu \mathrm{m}$ ). They form aggregations as presented with the arrows, with a mean maximum height of approximately $659 \mathrm{~nm}$, B) Platelets on type $\mathrm{A}$ carbon nanocoatings with $20 \% \mathrm{H}_{2}$ in plasma during deposition, after 2 hours of incubation (scan size $21 \mu \mathrm{m} \times 21 \mu \mathrm{m}$ ). The platelet clusters as denoted by the circles, have a height of more than $1000 \mathrm{~nm}$. 


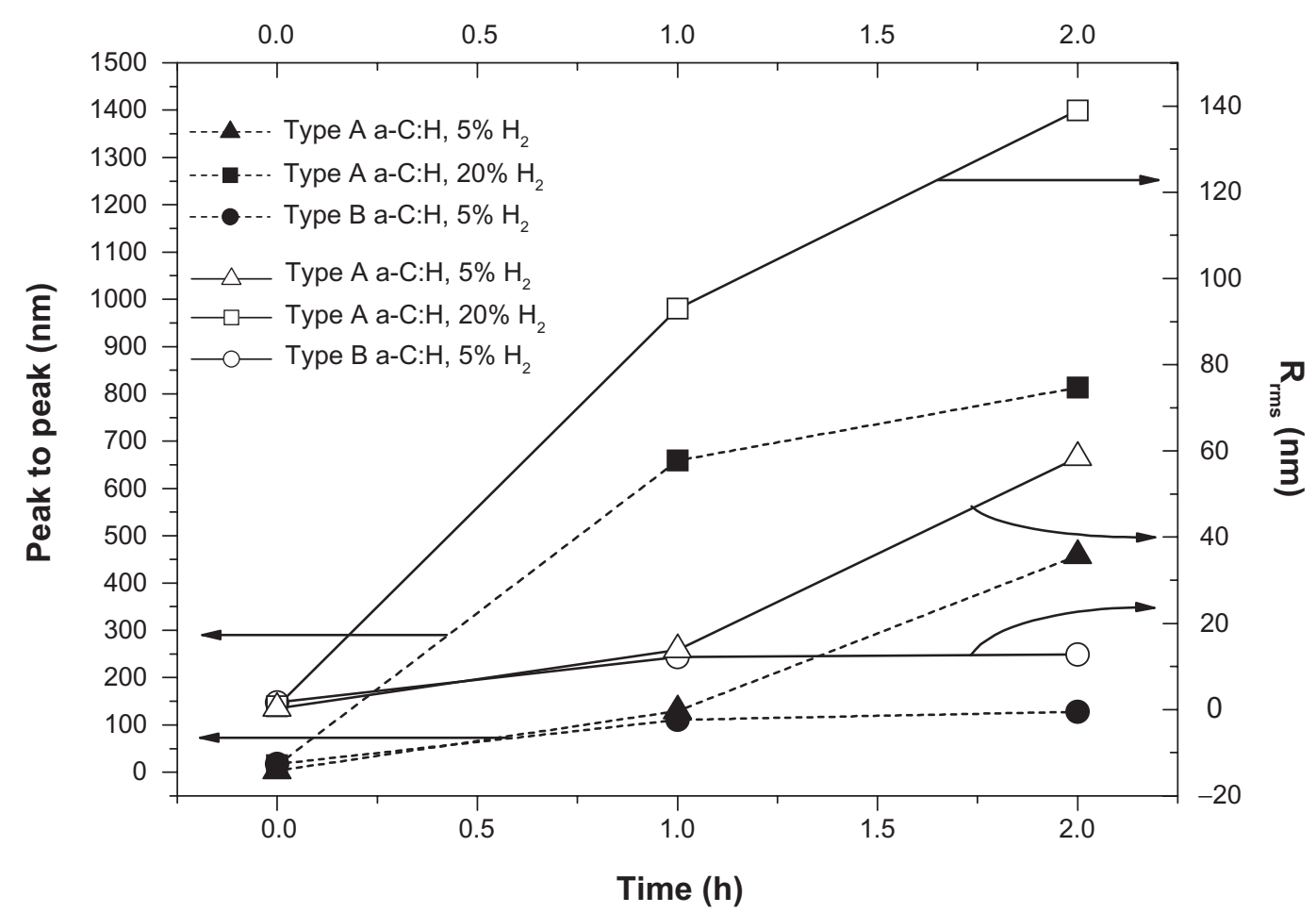

Figure 7 Comparative diagram of peak-to-peak parameter and root mean square roughness of platelet clusters versus incubation time for the studied types $A$ and $B$ carbon nanocoatings.

are more activated as they lose their spherical shape and increase their size, but still without forming clusters. These results are in accordance with the AFM images of the same samples after 1 hour of incubation (Figures $4 \mathrm{a}$ and $5 \mathrm{a}$ ).

\section{Discussion}

The state-of-the-art in DES technology is mainly the production of stents that elicit a cytotoxic or cytostatic drug that inhibits smooth muscle cells proliferation of the arterial wall and extra-cellular matrix targeting to these cells in order to halt the restenosis cascade. As a result, long-term artery restenosis rate after DES stent implantation is reduced. ${ }^{24-26}$ However, there are still concerns about the very late stent thrombosis of DES observed after 12 months of implantation, compared with bare metal stents. ${ }^{27}$ Although the cumulative incidence of stent thrombosis with DES at 9 to 12 months has ranged from $0.5 \%$ to $0.7 \%$ in clinical trials, roughly comparable to the incidence with bare metal stents, after 12 months, this pattern of thrombotic events was frequently observed beyond 12 months with DES but not with bare metal ones. ${ }^{28}$ This complication of DES was attributed mainly to inadequate endothelialization due to polymer coatings or elusion of the antiproliferative drug. Thus, the stent surface treatment by the design of biocompatible novel nanocoatings and the in-depth knowledge of the cells-biomaterials interaction with nanoscale precision are two of the challenges of nanotechnology. This is an interdisciplinary scientific field which is considered to be the key technology of the 21 st century, focusing on novel methods and materials, and tools with nanometer precision.

A number of nanotechnology approaches have been developed in stent technology. ${ }^{29,30}$ Stents with surface nanopores or nanoparticles with high stability and carrier capacity, and capable of incorporating both hydrophilic and hydrophobic substances in order to be used as carriers for bioactive factors and drugs have been manufactured. Many attempts have already been made to develop biodegradable or tissue-engineered and bioactive stents, which promote healing reactions by triggering the body's natural processes. ${ }^{31}$ Tissue engineering will enable biofunctionalized stents to create a biomimicking environment that promotes the desired endothelial cell activities, by exchanging the appropriate stimuli, and so promote their binding to specific endothelial membrane receptors.

Until now there has been a clear lack of data on the thrombogenicity of stent materials dictated by nanoscale observations. As thrombogenicity is a multiparametric process, the present work tested the hypothesis on the influence of surface nanotopography on platelets activation in order 
A

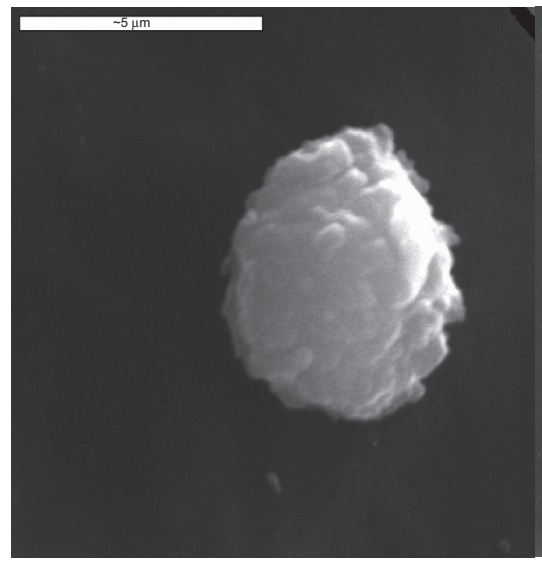

B

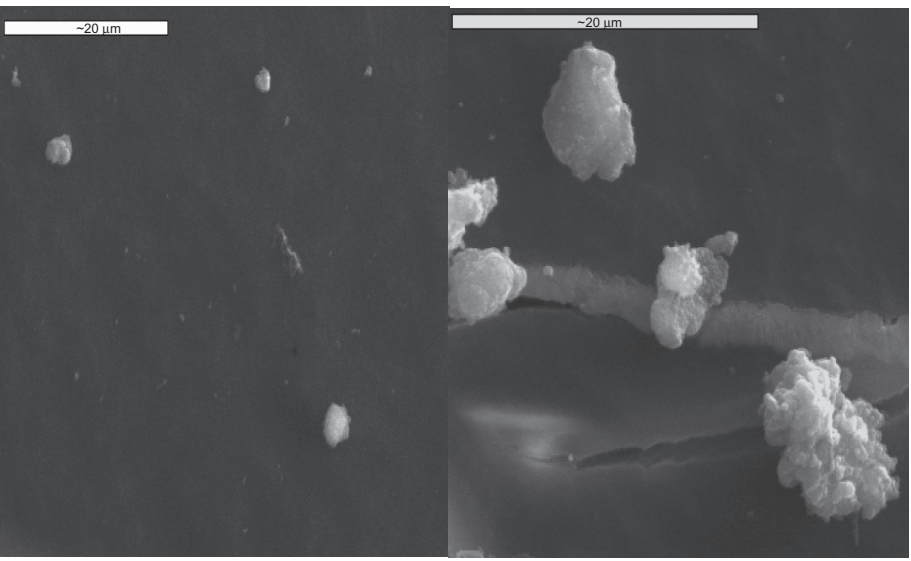

Figure 8 SEM images of: A) a human platelet onto carbon substrate, B) inactivated platelets on type $B$ carbon nanocoating with $5 \% \mathrm{H}_{2}$ in plasma during deposition, after one hour of incubation and $\mathbf{C}$ ) activated platelets on to type $A$ carbon nanocoating with $5 \% \mathrm{H}_{2}$ in plasma during deposition, after I hour of incubation.

to manufacture less thrombogenic stent nanocoatings by tailoring their surface properties. AFM, also called the 'eye of nanotechnology', was implemented to give evidence of a real time study of platelets response towards biomaterials for enhancing hemocompatibility.

Since proteins and cells range in size from nano- to micrometer scales, these length scales should be observed by an imaging tool able to observe and manipulate molecular and atomic level features and which can be used for highresolution and real-time studies of bio- and non-bio interactions. Until now, platelets studies were based on conventional biological imaging methods, such as SEM, which requires special preparation of the cells with gold and does not guarantee their viability. Therefore, a nondestructive, nanoscale precision technique for platelets study is needed, which can observe platelets in a native, unlabeled state for several hours without damage. One method that satisfies these demands is the AFM. ${ }^{32}$

An early event in blood-material interaction is the rapid and selective protein adsorption by a three-step process: the transport to the interface, the reaction of adsorption, and the conformational rearrangement. In addition, the implantassociated protein adsorption and conformational changes have been shown to promote immune reactions, and the engineering of surface properties (physical and chemical characteristics) has been rigorously researched in order to reduce protein adsorption and cell interactions and subsequently improve implant biocompatibility. ${ }^{33}$

Previous studies in our laboratory have focused on protein adsorption on carbon thin films measured by AFM and spectroscopic ellipsometry techniques. ${ }^{34-36}$ Cells adhesion occurs at a later stage and is mediated by the proteins initially adsorbed on the surface. Platelets, of the other cells present in blood, have the most important role in bloodmaterial interactions. A knowledge of basic cell-material interaction and an understanding of processes at the cellular level can aid in the development of new biomaterials with properties that can be prescribed and regulated. ${ }^{37}$ Therefore, there is a need to accumulate data on the interrelationship of physicochemical phenomena at biomaterial interfaces and their hemocompatible properties. The likelihood of the emergence of thrombosis caused by a biomaterial and especially the way that platelets react during their adhesion on to biomaterials, whether or not they activate the degree of activation and aggregation, are important parameters for hemocompatibility assessment, as proposed by ISO guidelines. ${ }^{38}$ Nevertheless, device-stent failure and/or other tissue-biomaterials interactions frequently cause clinically observable complications such as acute coronary syndrome, and necessitate reoperations or may be fatal. These deleterious outcomes may follow many years of uneventful benefit to the patient. Thus, there is a need for nanotechnology to contribute to the enhancement of thrombo-protectivity of stent nanocoatings by tailoring their properties on the basis of monitoring nanoscale platelet-surface interactions.

Platelet adhesion to a surface is governed by two independent mechanisms: the transport of platelets to the surface, which depends on the flow conditions, and the reaction of platelets with the surface, which depends on the nature of the surface and the adsorbed proteins. Platelet response after the contact of blood with an artificial surface is also influenced by diffusion, and intermolecular and shear forces. Platelets aggregation as described in this work may be attributed at first to the previous protein adsorption of PRP and the interaction 
Table I Surface roughness analysis of different types of bare carbon nanocoatings, including the mean values of peak-to-peak distance, root mean square roughness $\left(R_{r m s}\right)$ and their standard deviation values

\begin{tabular}{lll}
\hline $\begin{array}{l}\text { Roughness/ } \\
\text { Film type }\end{array}$ & $\begin{array}{l}\text { Mean peak-to- } \\
\text { peak }(\mathbf{n m})\end{array}$ & $\begin{array}{l}\text { Mean } \mathbf{R}_{\text {rms }} \\
\text { roughness }(\mathbf{n m})\end{array}$ \\
\hline Type A: a-C:H and $5 \% \mathrm{H}_{2}^{* * * *}$ & $3.2 \pm 0.2$ & $0.29 \pm 0.04$ \\
Type A: a-C:H and $20 \% \mathrm{H}_{2}$ & $14.9 \pm 1.3$ & $0.7 \pm 0.2$ \\
Type B: a-C:H and $5 \% \mathrm{H}_{2}$ & $16.8 \pm 1.6$ & $1.68 \pm 0.5$ \\
\hline
\end{tabular}

of platelets with the adsorbed fibrinogen or $\gamma$-globulin due to the formation of a complex between glycosyltransfereases located in the platelet membrane and incomplete heterosaccharides in the protein layer. Secondly, platelets connect with each other via filopods, and fibrinogen linkage with platelets surface receptors II $\beta$-IIIa, mediated by ATP and calcium. The formation of platelet islands may also be an outcome of intermolecular interactions, van der Waals, chemical, electrostatic forces (cell surface charge due to the content of sialic acid into their glycocalyx), and thermodynamic conditions involving the minimization of enthalpy and entropy.

In this work, we verified that nanotopography and surface characteristics influence the thrombogenicity of different types of carbon nanocoatings developed with magnetron sputtering, and that by tuning deposition parameters, thrombogenic behavior of the nanocoatings can be changed. All the aforementioned quantitative and qualitative data (variations in time-dependent platelets' surface roughness and AFM images, respectively) show that the rougher type B carbon nanocoatings activate the platelets less than the smoother type A carbon nanocoatings, whereas the content of $\mathrm{H}_{2}$ in plasma during deposition affects hydrogen configuration, surface properties, carbon bonding and subsequently their biological behavior. The above AFM results were compared, validated and verified by SEM results.

Also, these findings are in accordance with our hemocompatibility studies on the protein adsorption and platelets adhesion on biomaterials, which have shown that the nanotopography and surface roughness of biomaterials influence their biological behavior. Specifically, the carbon thin films with higher surface roughness were found to be more hemocompatible as they exhibit higher albumin fibrinogen adsorption ratio as measured by spectroscopic ellipsometry, ${ }^{39,40}$ and a lower degree of platelets activation. ${ }^{30,36}$ Recent studies on diamond like carbon (DLC) stent coatings suggest that the smooth surface of stent nanocoatings may promote thrombosis and may not encourage re-endothelialisation and that the surface roughness is correlated with the antithrombogenicity of DLC films. ${ }^{41,42}$ Another study suggests that surface roughness of biomaterials is a key factor in influencing thrombogenicity, and the evaluation of the fluorine doping in DLC films showed significant reductions in platelet adhesion and activation compared with DLC films for every grade of roughness. ${ }^{43}$

The hemocompatibility of an artificial material is an extremely complex and multiparametric process that it determines the efficacy of implants. Besides nanotopography ${ }^{44}$ and stoichiometry, we have found that wettability, and chemistry and surface electrical properties can also influence the fate of implants in the human body. ${ }^{45,46}$

\section{Conclusions}

This study has shown that the surface nanotopography of the stent nanocoating influences platelets behavior, which is a key factor for biomaterial thrombogenicity. Among the studied carbon nanocoatings those with the higher surface roughness value were found to be less thrombogenic in terms of platelets adhesion. Thus, by controlling the deposition conditions of the stent nanocoatings, the desirable surface properties that enhance their biological performance can be achieved. This is an example of how nanomedicine can revolutionize stent technology to meet the future challenge of late stent thrombosis, which is Achilles' heel of vascular stents.

\section{Acknowledgments}

The authors would like to thank Pr. E. Pavlidou for her help with SEM characterization.

\section{Disclosures}

The authors disclose no conflicts of interest.

\section{References}

1. Mukherjee D, Moliterno DJ. Effectiveness of drug-eluting stents in real-world patients. JAMA. 2008;299:454-455.

2. Jaffe RMD, Strauss BH. Late and very late thrombosis of drug-eluting stents. evolving concepts and perspectives. J Am Coll Cardiol. 2007; 50:1-9.

3. Stone GW, Moses JW, Ellis SG, et al. Safety and efficacy of sirolimus and paclitaxel-eluting coronary stents. N Engl J Med. 2007;356:998-1008.

4. Daemen J, Wenaweser P, Tsuchida K, et al. Early and late coronary stent thrombosis of sirolimus-eluting and paclitaxel-eluting stents in routine clinical practice: data from a large two-institutional cohort study. Lancet. 2007;369:667-678.

5. Iakovou I, Schmidt T, Bonizzoni E, Ge L, Sangiorgi GM, Stankovic G, et al. Incidence, predictors, and outcome of thrombosis after successful implantation of drug-eluting stents. JAMA. 2005;293:2126-2130.

6. Smith EJ, Jain KA, Rothman MT. New developments in coronary stent technology. J Intervent Cardiol. 2006;19(6):433-499.

7. Nebeker JR, Virmani R, Bennett CL, et al. Hypersensitivity cases associated with drug-eluting coronary stents: a review of available cases from the Research on Adverse Drug Events and Reports (RADAR) project J Am Coll Cardiol. 2006;47:175-181. 
8. Kathuria YP. The potential of biocompatible metallic stents and preventing restenosis. Mater Sci Eng A Struct Mater. 2006;417:40-48.

9. Miller DC, Thapa A, Haberstroh KM, Webster TJ. Endothelial and vascular smooth muscle cell function on poly(lactic-co-glycolic acid) with nano-structured surface features. Biomaterials. 2004;25:53-61.

10. Miller DC, Haberstroh KM, Webster TJ. Mechanism(s) of increased vascular cell adhesion on nanostructured poly(lactic-co-glycolic acid) films. J Biomed Mater Res A. 2005;73:476-484.

11. Caves J, Chaikof E. The evolving impact of microfabrication and nanotechnology on stent design. J Vasc Surg. 2006;44:1363-1368.

12. Liua H, Webster TJ. Nanomedicine for implants: A review of studies and necessary experimental tools. Biomaterials. 2007;28:354-369.

13. Dearnaley G, Arps JH. Biomedical applications of diamond-like carbon (DLC) coatings: A review. Surf Coat Technol. 2005;200:2518-2524.

14. Maguire D, McLaughlin J, Okpalugo T, et al. Mechanical stability, corrosion performance and bioresponse of amorphous diamond-like carbon for medical stents and guide wires. Diam Rel Materials. 2005;4:1277-1288.

15. Müller DJ, Anderson K. Biomolecular imaging using atomic force microscopy. Trends in Biotechnology. 2002;20:45-49.

16. Braet F, Seynaeve C, De Zanger R, et al. Imaging surface and submembranous structures with the atomic force microscope: a study on living cancer cells, fibroblasts and macrophages. J Microscopy. 1998;190: 328-338.

17. Mc Namee C, Pyo N, Tanaka S, et al. Imaging of a soft, weakly adsorbing, living cell with a colloid probe tapping atomic force microscope technique. Col and Surf B: Biointerfaces. 2006;47:85-89.

18. Okpalugo T, Ogwu A, Maguire P, et al. In-vitro blood compatibility of a-C:H:Si and a-C:H thin films. Diam Relat Mater. 2004;13:1088-1092.

19. Logothetidis S, Gioti M, Lousinian S, et al. Haemocompatibility studies on carbon-based thin films by ellipsometry. Thin Solid Films 2005;482:126-132.

20. Lousinian S, Logothetidis S. Optical properties of proteins and protein adsorption study. Microelect Engin. 2007;84:479-485.

21. Roya RK, Choia HW, Yia JW, et al. Hemocompatibility of surfacemodified, silicon-incorporated, diamond-like carbon films. Acta Biomaterialia. 2009;5:249-256.

22. Fritz M, Radmacher M, Gaub HE. Granula motion and membrane spreading during activation of human platelets imaged by atomic force microscopy. Biophys J. 1994;66:1328-1334.

23. Hartwig JH. Platelet structure. In: Michelson AD, editor. Platelets. Academic Press; 2002;37-45.

24. Vijayanand K, Deepak K. Pattanayak D, et al. Interpreting bloodbiomaterial interactions from surface free energy and work of adhesion. Trends Biomater Artif Organs. 2005;18:73-83.

25. Stone GW, Ellis SG, Cannon L, et al. TAXUS V Investigators. Comparison of a polymer-based paclitaxel-eluting stent with a bare metal stent in patients with complex coronary artery disease: A randomized controlled trial. JAMA. 2005;294:1215-1223.

26. Ong AT, van Domburg RT, Aoki J, et al. Sirolimus-eluting stents remain superior to bare-metal stents at two years: Medium-term results from the Rapamycin-Eluting Stent Evaluated at Rotterdam Cardiology Hospital (RESEARCH) registry. J Am Coll Cardiol. 2006;47:1356-1360.

27. Valgimigli M, Malagutti P, Aoki J, et al. Sirolimus-eluting versus paclitaxel-eluting stent implantation for the percutaneous treatment of left main coronary artery disease: A combined RESEARCH and T-SEARCH long-term analysis. J Am Coll Cardiol. 2006;47:507-514.
28. Joner M, Finn AV, Farb A, et al. Pathology of drug-eluting stents in humans: delayed healing and late thrombotic risk. J Am Coll Cardiol. 2006:48:193-202

29. Kaul S, Shah P, Diamond G. As time goes by current status and future directions in the controversy over stenting. JAm Coll Cardiol. 2007;50: $1-10$.

30. Karagkiozaki V, Logothetidis S, Giannoglou G. Advances in stent coating technology via nanotechnology tools and process. Eur $J$ Nanomedicine. 2008;1:24-28.

31. William D. Knopf W. The clinical performance and angiographic results of the Coronary Stenting and Absorbable Metal Stents Study. PROGRESS AMS. J Am Coll Cardiol. 2006;47:9-12.

32. Radmacher M, Tillamnn R, Fritz M, Gaub HE. From molecules to cells: imaging soft samples with the atomic force microscope. J Science. 1992;257:1900-1905.

33. Tang L, Thevenot P, Hu W. Surface chemistry influences implant biocompatibility. Current Top Med Chem. 2008;8:270-280.

34. Lousinian S, Logothetidis S. In-situ and real-time protein adsorption study by Spectroscopic Ellipsometry. Thin Solid Films. 2008;516:8002-8008.

35. Logothetidis S. Haemocompatibility of carbon based thin films. Diam Relat Mater. 2007;16:1847-1857.

36. Karagkiozaki V, Logothetidis S, Laskarakis A, et al. AFM Study of the thrombogenicity of carbon-based coatings for cardiovascular applications. Mater Sci Engineer B. 2008;152:16-21.

37. Yang P, Huang N, Leng YX, et al. Activation of platelets adhered on amorphous hydrogenated carbon films synthesized by plasma immersion ion implantation-deposition. Biomaterials. 2003;24:2821-2829.

38. ISO 10993-4: 2002, Selection of Tests for Interactions with Blood.

39. Mitsakakis K, Lousinian S, Logothetidis S. Early stages of human plasma proteins adsorption probed by Atomic Force Microscope. Biomol Engineer. 2007;24:119-124.

40. Lousinian S, Kalfagiannis N, Logothetidis S. Albumin and fibrinogen adsorption on boron nitride and carbon-based thin films. Mater Sci and Engineer B. 2008;152:12-15.

41. Seliger C, Schwennicke K, Schaffer C, et al. Influence of a rough ceramic-like stent surface made of iridium oxide on neointimal structure and thickening. Euro Heart J. 2002;21:282-290.

42. Ong SE, Zhang S, Du H, Too HC, Aung KN. Influence of silicon concentration on the haemocompatibility of amorphous carbon. Biomaterials. 2007;28:4033-4038.

43. Hasebe T, Ishimaru T, Kamijo A, et al. Effects of surface roughness on anti-thrombogenicity of diamond-like carbon films. Diam and Rel Mater. 2007;16:1343-1348

44. Karagkiozaki V, Logothetidis S, Kassavetis S, Lousinian S. Nanoscale characterization of biological and mechanical profile of carbon stent nanocoatings. Eur J Nanomedicine. 2009;2:14-21.

45. Karagkiozaki V, Logothetidis S, Kalfagiannis N, et al. AFM probing platelets activation behavior on titanium nitride nanocoatings for biomedical applications. Nanomedicine. 2009;5:64-72.

46. Karagkiozaki V, Logothetidis S, Lousinian S, et al. Impact of surface electric properties of carbon-based thin films on platelets activation for nano-medical and -sensing applications. Int $J$ Nanomedicine. 2008:3:461-469.
International Journal of Nanomedicine

\section{Publish your work in this journal}

The International Journal of Nanomedicine is an international, peerreviewed journal focusing on the application of nanotechnology in diagnostics, therapeutics, and drug delivery systems throughout the biomedical field. This journal is indexed on PubMed Central, MedLine, CAS, SciSearch ${ }^{\circledR}$, Current Contents ${ }^{\circledR} /$ Clinical Medicine,

\section{Dovepress}

Journal Citation Reports/Science Edition, EMBase, Scopus and the Elsevier Bibliographic databases. The manuscript management system is completely online and includes a very quick and fair peer-review system, which is all easy to use. Visit http://www.dovepress.com/ testimonials.php to read real quotes from published authors. 